\title{
Aplicação do escore CRIB para avaliar $o$ risco de mortalidade neonatal
}

\author{
The use of CRIB score for predicting neonatal mortality risk
}

\author{
Ana Lúcia F. Sarquis ${ }^{1}$, Mitsuru Miyaki ${ }^{2}$, Mônica N.L. Cat ${ }^{3}$
}

\section{Resumo}

Objetivos: 1) avaliar o valor preditivo do Clinical Risk Index for Babies (CRIB) para óbito hospitalar; 2) identificar a variável do escore com melhor valor preditivo; e 3) comparar a capacidade do escore CRIB para predizer mortalidade hospitalar com a do peso de nascimento, da idade gestacional e do excesso de base isolados.

Métodos: o escore CRIB foi aplicado de forma prospectiva em 100 recém-nascidos admitidos consecutivamente na Unidade Neonatal do HC-UFPR, que tinham peso de nascimento igual ou inferior a $1.500 \mathrm{~g}$ ou idade gestacional menor que 31 semanas.

Resultados: cinqüenta e cinco recém-nascidos eram do sexo feminino e 45, do masculino, a média do peso de nascimento foi de $1.078,0 \pm 277,0 \mathrm{~g}$, e da idade gestacional de 29,2 $\pm 2,8$ semanas. Vinte e um pacientes foram a óbito. A mortalidade nos graus 1, 2, 3 e 4 do CRIB foi, respectivamente, de 6,6\%; 46,2\%, 85,7\% e 100,0\%. A precisão do escore para mortalidade foi confirmada (área sob a curva $\mathrm{ROC}=0,877$ ), e a melhor variável do escore para prognosticar o óbito hospitalar foi o excesso de base máximo (área sob a curva $\mathrm{ROC}=0,795)$. Comparado com peso de nascimento e idade gestacional, o CRIB foi significativamente melhor para predizer mortalidade.

Conclusões: além de ser útil no prognóstico do óbito hospitalar, o CRIB mostrou-se um escore de aplicação simples. Com base nos resultados encontrados, recomenda-se sua incorporação na rotina das unidades neonatais.

J Pediatr (Rio J) 2002; 78 (3): 225-9: recém-nascido prematuro, mortalidade neonatal, escore de gravidade clínica.

\section{Introdução}

Quando se analisa a perspectiva de sobrevida de recémnascidos internados numa UTI neonatal, a que desperta maior preocupação é, sem dúvida, a do recém-nascido

\footnotetext{
1. Mestre em Pediatria (UFPR), Professora Substituta de Neonatologia UFPR.

2. Mestre em Pediatria (UFPR), Professor Titular de Pediatria - UFPR

3. Mestre em Pediatria (UFPR), Professora Assistente de Neonatologia UFPR.

Artigo submetido em 19.10.01, aceito em 13.03.02.
}

\begin{abstract}
Objective: to examine the clinical risk index for babies (CRIB) predictive value for hospital death; to identify the score variable with the best predictive value and to compare CRIB score capability to predict hospital mortality to birth weight, gestational age and base excess.

Methods: CRIB score was obtained through a prospective way from 100 newborns with birthweight of $1,500 \mathrm{~g}$ or less or gestational age less than 31 weeks, who were admitted consecutively to the Neonatal Unit of Hospital das Clínicas, Universidade Federal do Paraná.

Results: Fifty-five newborns were female and 45 were male, the average of birthweight was $1,078 \pm 0.277 \mathrm{~g}$ and gestational age was $29.2 \pm 2.8$ weeks. Twenty-one patients died. The mortality rate in the CRIB groups 1, 2, 3 and 4 was, respectively $6.6 \% ; 46.2 \% ; 87.5 \%$ and $100.0 \%$. The score accuracy for mortality was confirmed (area under the ROC curve $=0.877$ ) and the best score variable to predict hospital death was maximum base excess (area under the ROC curve = 0.795). Compared with birthweight and gestational age, CRIB was significantly better to predict mortality.

Conclusions: Besides being useful to predict hospital death, CRIB was a simple score to be applied. Based on these results, we recommend its inclusion in the routine of neonatal units.
\end{abstract}

J Pediatr (Rio J) 2002; 78 (3): 225-9: preterm infant, neonatal mortality, illness severity score.

prematuro ${ }^{1,2}$. A assistência médica com qualidade adequada nos cuidados neonatais, tanto quanto a disponibilidade de recursos, é fundamental, principalmente quando se trata de prematuros com peso de nascimento menor que 1.500 gramas ou de idade gestacional menor que 31 semanas $^{1}$. O risco de óbito destes prematuros, por ser muito elevado, torna-os um grupo particular para estudo de avaliação de desempenho das UTI neonatais ${ }^{1}$. Alguns escores de avaliação vêm sendo propostos para analisar de forma mais 
objetiva a perspectiva de sobrevivência e a qualidade de vida dos prematuros e, entre eles, destaca-se o Clinical Risk Index for Babies (CRIB) ${ }^{1}$. Trata-se de um escore que avalia a gravidade clínica inicial em recém-nascidos prematuros com base no peso de nascimento, idade gestacional, malformação congênita, excesso de base e fração inspirada de oxigênio (Tabela 1). Foi desenvolvido pelo International Neonatal Network no Reino Unido, em 1993, e é útil para medir o risco de óbito hospitalar ${ }^{1}$.

Este estudo teve como objetivos:

- avaliar o valor preditivo do CRIB para óbito hospitalar em nosso meio;

- identificar a variável do escore com melhor valor preditivo; e

- comparar a capacidade do escore CRIB, da idade gestacional e do peso de nascimento para predizer mortalidade hospitalar.

\section{Métodos}

O presente estudo foi desenvolvido como método prospectivo, descritivo e observacional, e envolveu os prematuros admitidos na UTI-Neonatal do Hospital de Clínicas da Universidade Federal do Paraná, no período compreendido entre $1^{\circ}$ de maio de 1998 e 11 de junho de 1999 . Foram obedecidos os critérios de inclusão preconizados pelo International Neonatal Network ${ }^{1}$, ou seja: peso de nascimento igual ou inferior a $1.500 \mathrm{~g}$, ou idade gestacional menor que 31 semanas. Foram excluídos do estudo os recémnascidos com malformações incompatíveis com a vida (anencefalia, trissomia do 18 e agenesia renal) ${ }^{1}$. Não foram incluídos os pacientes transferidos de outros serviços.

Cada variável do escore CRIB tem um valor numérico pré-determinado que varia conforme a gravidade (Tabela 1), e após obter os valores somados desses itens, os pacientes são classificados em 4 graus: grau 1 para escores de 0 a 5, grau 2 de 6 a 10, grau 3 de 11 a 15, e grau 4 para pontuações maiores do que 15. O escore CRIB foi aplicado nas primeiras 12 horas de vida, com base na evolução médica, tendo sido anotados os valores extremos da $\mathrm{FiO}_{2}$, que foram utilizados, e o valor mais elevado do BE obtido pela gasometria arterial. Foi considerada $\mathrm{FiO}_{2}$ apropriada aquela necessária para manter uma saturação de oxigênio da hemoglobina entre 90 e $96 \%$ pelo oxímetro de pulso.

A idade gestacional definitiva foi estimada: 1) pela data da última menstruação (cronológica), quando a gestante tinha ciclos menstruais regulares e não estava em uso de anticoncepcionais orais no momento da concepção; 2) pelo método de Ballard, modificado para inclusão de pequenos prematuros $^{2}$, se houvesse dúvida na cronologia da gestação; ou 3) através de ultra-sonografia, no caso de idade gestacional cronológica não confiável ou impossibilidade da realização do exame clínico.

O acompanhamento de cada paciente foi realizado até a ocasião da alta hospitalar, com registro dos óbitos ocorridos.
Tabela 1 - Escore CRIB

\begin{tabular}{|c|c|}
\hline Variável & Pontuação \\
\hline \multicolumn{2}{|l|}{ Peso de nascimento $(\mathrm{g})$} \\
\hline$>1.350$ & 0 \\
\hline $851-1.350$ & 1 \\
\hline $701-850$ & 4 \\
\hline$\leq 700$ & 7 \\
\hline \multicolumn{2}{|l|}{ Idade gestacional (semanas) } \\
\hline$>24$ & 0 \\
\hline$\leq 24$ & 1 \\
\hline \multicolumn{2}{|l|}{ Malformação congênita } \\
\hline Ausente & 0 \\
\hline Sem risco de vida imediato & 1 \\
\hline Com risco de vida imediato & 3 \\
\hline \multicolumn{2}{|c|}{ BE máximo nas primeiras $12 \mathrm{~h}$ de vida (mmol/l) } \\
\hline$>-7,0$ & 0 \\
\hline$-7,0$ a $-9,9$ & 1 \\
\hline$-10,0$ a $-14,9$ & 2 \\
\hline$\leq-15,0$ & 3 \\
\hline \multicolumn{2}{|c|}{$\mathrm{FiO}_{2}$ mínima apropriada nas primeiras $12 \mathrm{~h}$ de vida } \\
\hline$\leq 0,40$ & 0 \\
\hline $0,41-0,60$ & 2 \\
\hline $0,61-0,90$ & 3 \\
\hline $0,91-1,00$ & 4 \\
\hline \multicolumn{2}{|c|}{$\mathrm{FiO}_{2}$ máxima apropriada nas primeiras $12 \mathrm{~h}$ de vida } \\
\hline$\leq 0,40$ & 0 \\
\hline $0,41-0,80$ & 1 \\
\hline $0,81-0,90$ & 3 \\
\hline $0,91-1,00$ & 5 \\
\hline
\end{tabular}

Para a análise dos dados, foram utilizados o teste $\mathrm{t}$ de Student, o teste do Qui-quadrado de Pearson e o teste exato de Fischer. A análise de especificidade e sensibilidade das variáveis estudadas para predição de óbito foi realizada através de curvas ROC, sendo que, para discriminar as diferenças com significância estatística, as áreas sob as curvas foram comparadas pelo método de Hanley, Mc$\mathrm{Neil}^{3}$.

Este estudo foi aprovado pelo Comitê de Ética do HCUFPR.

\section{Resultados}

No período do estudo, foram internados 620 recémnascidos, e 100 preencheram os critérios de inclusão e constituíram o grupo de estudo.

Cinquienta e cinco recém-nascidos eram do sexo feminino, e o restante do masculino.

As médias de peso de nascimento e idade gestacional nos pacientes que foram a óbito e nos que sobreviveram, bem como os respectivos desvios-padrões, valores mínimos e máximos, encontram-se na Tabela 2. 
Tabela 2 - Média, valor mínimo, máximo e desvio-padrão (DP) do peso de nascimento e da idade gestacional nos sobreviventes e nos pacientes que foram a óbito

\begin{tabular}{lcccc}
\hline & \multicolumn{2}{c}{ Peso de nascimento $(\mathbf{g})$} & \multicolumn{2}{c}{ Idade gestacional (semanas) } \\
& Óbito & Sobrevivente & Sobrevivente \\
\hline Média & $942,14^{*}$ & $1.213,8$ & $27,7^{*}$ & 30,6 \\
Mínimo & 495,0 & 565,0 & 23,3 & 26,0 \\
Máximo & $1.460,0$ & $1.810,0$ & 32,4 & 37,0 \\
DP & 302,4 & 241,5 & 3,0 & 2,5 \\
\hline
\end{tabular}

${ }^{*} p<0,01$

Nove por cento dos pacientes apresentavam malformações congênitas.

A taxa de mortalidade hospitalar foi de $21,0 \%$. A pontuação média do escore CRIB foi de 4,0 $\pm 4,6$, com mediana de 2,0 e variação de 0 a 19 pontos. Os recémnascidos foram divididos em 4 faixas crescentes de gravidade, conforme preconizado pelos autores do CRIB $^{1}$, e a mortalidade correspondente a cada grau foi comparada, obtendo-se uma associação linear estatisticamente significativa (Tabela 3).

Tabela 3 - Distribuição dos recém-nascidos quanto aos graus do CRIB e respectiva mortalidade

\begin{tabular}{lccc}
\hline Classificação & N & $\%$ & Mortalidade \\
\hline Grau 1 & 76 & 76,0 & $5(6,6 \%)^{*}$ \\
Grau 2 & 13 & 13,0 & $6(46,2 \%)$ \\
Grau 3 & 7 & 7,0 & $6(85,7 \%)$ \\
Grau 4 & 4 & 4,0 & $4(100,0 \%)$ \\
\hline
\end{tabular}

${ }^{*} \mathrm{p}<0,01$

Na Figura 1, está representada a curva ROC do escore CRIB, sendo o valor sob a curva de 0,877 (IC 95\%: 0,797 - 0,934) com erro padrão de 0,051. A curva encontra-se desviada para cima e para a direita, localizando-se no canto superior direito do gráfico, o que significa que, à medida que a sensibilidade diminui, esta não se acompanha de perda da especificidade, caracterizando-o portanto como um bom teste discriminatório.

$\mathrm{Na}$ Tabela 4, encontram-se os valores da área sob a curva ROC, erro padrão e IC (95\%) do escore CRIB e das variáveis $\mathrm{BE}$, peso de nascimento e idade gestacional isoladamente. Observou-se que o maior valor da área sob a curva ROC foi do escore CRIB, seguido, em ordem decrescente, pelo BE, peso de nascimento e idade gestacional. A avaliação isolada das variáveis que compõem o escore CRIB evidenciou que o BE apresentou capacidade preditiva para o risco de óbito hospitalar semelhante ao do escore como um todo, enquanto o peso de nascimento e a idade gestacional isoladamente demonstraram esta habilidade em nível significativamente menor.

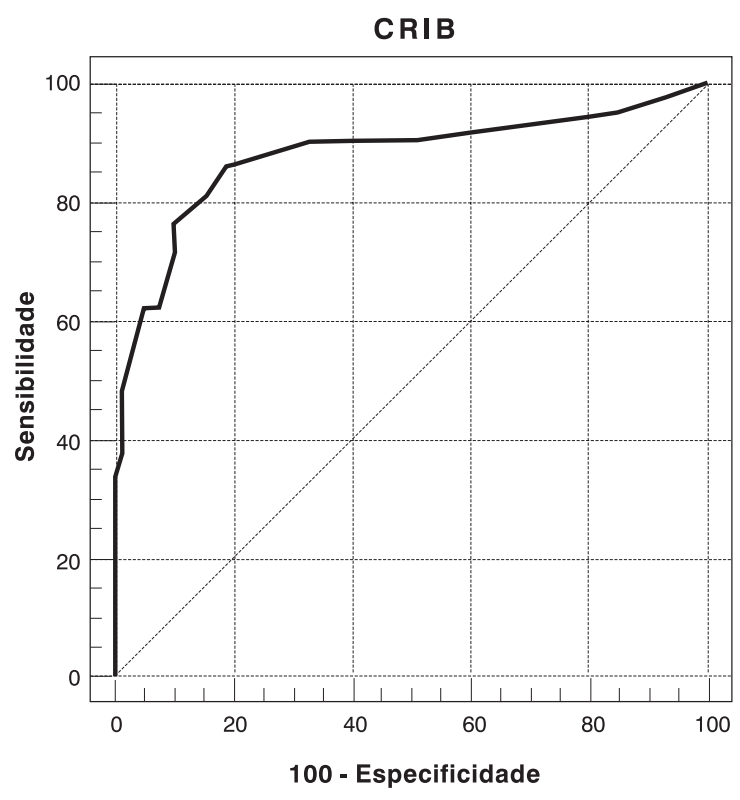

Figura 1 - Curva ROC do escore CRIB 
Tabela 4 - Valores da área sob a curva ROC, erro padrão, IC (95\%) do escore CRIB e das variáveis $\mathrm{BE}$, peso de nascimento e idade gestacional

\begin{tabular}{lcccc}
\hline Variável & $\begin{array}{c}\text { Área sob a } \\
\text { curva ROC }\end{array}$ & $\begin{array}{c}\text { Erro } \\
\text { padrão }\end{array}$ & IC 95\% & p \\
\hline Escore CRIB & 0,877 & 0,051 & $0,797-0,934$ & \\
BE & 0,795 & 0,048 & $0,621-0,805$ & 0,06 \\
Peso de nascimento & 0,754 & 0,053 & $0,658-0,843$ & 0,02 \\
Idade gestacional & 0,751 & 0,053 & $0,655-0,832$ & 0,02 \\
\hline
\end{tabular}

\section{Discussão}

$\mathrm{Na}$ atividade assistencial para os recém-nascidos internados num Serviço de Neonatologia, o grupo que exige maior atenção é o de prematuros e, destes, em especial, os grandes prematuros. Indiscutivelmente a sobrevivência destes pequenos pacientes não depende somente da qualidade e da intensidade dos cuidados neonatais disponibilizados, como também são importantes os graus de imaturidade e o peso de nascimento 5 .

Em relação à mortalidade observada, foi verificada a freqüência de $21,0 \%$, resultado este compatível com o de estudos nacionais e internacionais, desde que com população de estudo e recursos terapêuticos semelhantes ${ }^{6-8}$.

Durante a realização do presente estudo foi constatado que o escore CRIB foi de fácil aplicação. Ele foi um escore prático, pois utilizou variáveis que faziam parte da rotina de atendimento dos recém-nascidos prematuros, sendo sua obtenção efetuada rapidamente. Devido a sua simplicidade, o escore CRIB foi considerado também como sendo de fácil reprodução, não dando margem a erros de interpretação por subjetividade individual.

A expressão quantitativa do poder do escore como marcador de previsão de morte foi avaliada através da área que a curva determinou sobre o espaço delimitado pelo gráfico. Neste sentido, o escore CRIB confirmou sua capacidade preditiva para a ocorrência de óbito hospitalar, com área sob a curva ROC de 0,877 , resultado este muito semelhante ao do estudo que o originou, que foi de $0,90^{1}$, bem como aos de outros estudos: 0,83 no Scottish Neonatal Consultants' Study Group e International Neonatal Network ${ }^{9} ; 0,89$ por Rautonen et al. ${ }^{10} ; 0,90$ por Bastos et al. ${ }^{9}$; 0,93 por Baumer et al. ${ }^{2}$ e 0,90 por Matsuoka et al. ${ }^{8}$.

$\mathrm{Na}$ análise da comparação entre os 4 graus do escore CRIB para a ocorrência de óbito hospitalar, os resultados obtidos estão de acordo com a proposta do estudo original, ou seja, quanto maior o grau do escore, maior a ocorrência de óbito. Demonstrou-se uma relação significativa entre as categorias ascendentes do escore e o óbito dos recémnascidos prematuros, principalmente na comparação do grau 1 com os demais. Resultados análogos foram publicados em diversos estudos internacionais e nos dois estudos nacionais $1,2,7,8,10,12,13$.

No presente estudo, chamou a atenção o fato de apenas um paciente do grau 3 e nenhum do grau 4 ter sobrevivido. Estes grupos merecem especial atenção, necessitando esforços ainda maiores para se obter redução da sua mortalidade. Estes resultados são comparáveis aos da literatura nacional ${ }^{7,8}$; entretanto, no estudo que deu origem ao escore CRIB, a mortalidade para os pacientes com pontuações superiores a 10 não atingiu $80,0 \%{ }^{8}$.

A expressão quantitativa do poder de cada variável, como marcador de previsão de óbito, pode ser avaliada pela área que cada curva determina no espaço delimitado do gráfico $^{3}$. Neste sentido, o escore CRIB demonstrou sua superioridade significativa quando comparado com idade gestacional e peso de nascimento. Confirmou-se, neste estudo, que a idade gestacional e o peso de nascimento isolados devem ser substituídos por um conjunto de variáveis que leve em consideração também a avaliação da gravidade clínico-laboratorial dos recém-nascidos nas primeiras 12 horas de vida. Este resultado está de acordo com dados relatados na literatura ${ }^{1,2,9,12,13}$. O estudo de Zullini et al. ${ }^{7}$, contrariamente evidenciou que a eficácia preditiva do peso de nascimento isolado foi semelhante a do escore CRIB, com áreas sob a curva ROC de 0,810 e 0,800, respectivamente. A capacidade preditiva do escore CRIB no estudo de Matsuoka et al. ${ }^{8}$ determinada pela área sob a curva ROC foi de 0,900 , enquanto a do peso de nascimento foi de 0,830 .

Entre as variáveis do escore CRIB, o BE máximo, nas primeiras 12 horas de vida, foi a que teve melhor precisão para predizer o óbito hospitalar, com uma área sob a curva ROC igual a 0,795 . O excesso de base, observado nas primeiras horas de vida, pode ser considerado como um indicador inespecífico de acúmulo de ácidos, e este, como 
um sinal de instabilidade fisiológica aguda ao nascimento, e, indiretamente, portanto, pode refletir perfusão tecidual e oxigenação inadequadas. Seguindo este raciocínio, podese inferir que a maior capacidade do BE para determinar óbito hospitalar, quando comparada ao peso de nascimento e à idade gestacional, reforça a necessidade de considerar a gravidade clínica do paciente na avaliação do risco de mortalidade. $\mathrm{O}$ valor preditivo do BE para óbito em recémnascidos de muito baixo peso foi também demonstrado por outros autores, ao desenvolverem um escore quantitativo do risco de mortalidade neonatal no momento da admissão na UTI neonatal ${ }^{14}$. Estudos em adultos têm demonstrado que o excesso de base em sangue arterial é uma variável importante na ressuscitação de pacientes politraumatizados, e que a sua magnitude, no momento da admissão hospitalar, está significativamente relacionada ao desenvolvimento de disfunção de múltiplos órgãos e mortalidade. Resultados de um estudo recente, em adultos vítimas de trauma, demonstraram que a capacidade preditiva do BE para óbito após a admissão na UTI correspondeu a uma área sob a curva ROC de $0,710^{15}$.

O CRIB reúne as três propriedades necessárias para um bom escore preditivo de mortalidade: é confiável, de fácil aplicação e preciso ${ }^{16,17}$. Tanto pelo seu valor prognóstico, quanto pela sua facilidade de utilização, parece ser o escore de preferência a ser adotado para a avaliação do desempenho das unidades neonatais, em especial nos países em que os recursos diagnósticos e terapêuticos mais sofisticados são limitados.

\section{Referências bibliográficas}

1. International Neonatal Network. The CRIB (clinical risk index for babies) score: a tool for assessing initial neonatal risk and comparing performance of neonatal intensive care units. Lancet 1993; 342:193-8.

2. Baumer JH, Wright D, Mill T. Illness severity measured by CRIB score: a product of changes in perinatal care? Arch Dis Child 1997; 77:211-5.

3. Hanley JA, McNeil BJ. The meaning and use of the area under a Receiver Operating Characteristic (ROC) Curve. Radiology 1982; 143:29-36.

4. Ballard JL, Khoury JC, Wedig K, Wang L, Eilers-Walsman, Lipp R. New Ballard score, expanded to include extremely premature infants. J Pediatr 1991; 119:417-23.

5. Hope P. CRIB, son of Apgar, brother to APACHE. Arch Dis Child 1995; 72:F81-83.
6. Horbar JD, Onstad L, Wright E, The National Institute of Child Health and Human Development Neonatal Research Network. Predicting mortality risk for infants weighing 501 to 1,500 grams at birth: a National Institute of Health Neonatal Research Network report. Crit Care Med 1993; 21:12-8.

7. Zullini da Costa MTZ, Ventura GB, Melo AMG, Okay Y, Ramos JLA, Vaz FAC. CRIB escore: um instrumento para avaliar o risco de óbito em recém-nascidos prematuros. Pediatria (São Paulo) 1998; 20:255-60.

8. Matsuoka OT, Sadekc SR, Haber JFS, Proença RSM, Mataloun MMG, Ramos JLA, et al. Valor preditivo do "Clinical Risk Index for Babies" para o risco de mortalidade neonatal. Rev Saúde Pública 1998; 32:550-5.

9. Scottish Neonatal Consultants' Collaborative Study Group, International Neonatal Network. CRIB (clinical risk index for babies), mortality, and impairment after neonatal intensive care. Lancet 1995; 345:1020-2.

10. Rautonen J, Mälelä A, Boyd H, Apajasalo M, Pohjavuouri M. CRIB and SNAP: assessing the risk of death for preterm neonates. Lancet 1994; 343:1272-3.

11. Bastos G, Gomes A, Oliveira P, Torrado da Silva A. Comparação de quatro escalas de avaliação da gravidade clínica (CRIB, SNAP, SNAP-PE, NTISS) em recém-nascidos prematuros. Acta Med Port 1997; 10:161-5.

12. Courcy Wheeler RHB, Wolfe CDA, Fitzgerld A, Spencer M, Goodman JDS, Gamsu JR. Use of CRIB (clinical risk index for babies) score in prediction of neonatal mortality. Arch Dis Child 1995; 73:32-6.

13. Kaarsen PI, Dohlen G, Fundingsrud HP, Dahl IB. The use of CRIB (clinical risk index for babies) score in auditing the performance of one neonatal intensive care unit. Acta Paediatr 1998; 87:195-200.

14. Maier RF, Rey M, Metze BC, Obladen, M. Comparison of mortality risk: a score for very low birthweight infants. Arch Dis Child 1997; 76:F146-F151.

15. Kincaid EH, Miller PR, Meredith JW, Rahman N, Chang MC. Elevated arterial base deficit in trauma patients: a marker of impaired oxygen utilization. J Am Coll Surg 1998; 187:384-92.

16. Fleisher BE, Murthe L, Lee S, Constantinou JC, Benitz WE, Stevenson DK. Neonatal severity of illness scoring systems: a comparison. Clin Pediatr (Phila) 1997:223-7.

17. Fowlie PW, Gould CR, Tarnow-Mordi, WO, Strang D. Measurement properties of the clinical risk index for babies reliability, validity beyond the first 12 hours, and responsiveness over 7 days. Crit Care Med 1998; 26:163-8.

Endereço para correspondência:

Dra. Ana Lúcia Sarquis

Rua Emílio Cornelsen, 570 - apto. 704 B5 - Ahú

CEP 80540-220 - Curitiba, PR

Fone: (41) 254.5603

E-mail: anasarquis@terra.com.br 